\title{
Effects of Thermal Radiation and Radiation Absorption on Flow Past an Impulsively Started Infinite Vertical Plate with Newtonian Heating and Chemical Reaction
}

\author{
Swetha Ravi', Jagdish Prakash ${ }^{2}$, Viswanatha Reddy Gottam³ ${ }^{3}$ Vijaya Kumar Varma Sibyala ${ }^{3}$ \\ ${ }^{1}$ Department of Mathematics, Gudlavalleru Engineering College, Gudlavalleru, India \\ ${ }^{2}$ Department of Mathematics, University of Botswana, Gaborone, Botswana \\ ${ }^{3}$ Department of Mathematics, S. V. University, Tirupati, India \\ Email: swetha.salini@gmail.com, “prakashj@mopipi.ub.bw,gvr.svu@gmail.com, \\ svijayakumarvarma@yahoo.co.in
}

Received 3 November 2015; accepted 24 December 2015; published 29 December 2015

Copyright $@ 2015$ by authors and Scientific Research Publishing Inc.

This work is licensed under the Creative Commons Attribution International License (CC BY).

http://creativecommons.org/licenses/by/4.0/

(c) (i) Open Access

\section{Abstract}

A perfect solution to the present natural convective flow problem of a vertical transfinite plate owing to the impulsive motion in the ubiety of first ordered chemical reaction, radiation absorption, radiation, Newtonian heating and species concentration in its plane is evolved by applying the method of Laplace transforms in closed form at the plate. Exact results for velocity, temperature, concentration fields are prevailed and expressions for heat and mass transfer rates are also found. The effects are analyzed for the respective invariables for both ammonia and water vapor.

\section{Keywords}

Newtonian Heating, Natural Convection, Chemical Reaction, Incompressible Fluid, Radiation Absorption and Radiation

\section{Introduction}

On chemical reaction, the field of mass and heat transfer is of good pragmatic importance to applied scientists ${ }^{*}$ Corresponding author.

How to cite this paper: Ravi, S., Prakash, J., Gottam, V.R. and Sibyala, V.K.V. (2015) Effects of Thermal Radiation and Radiation Absorption on Flow Past an Impulsively Started Infinite Vertical Plate with Newtonian Heating and Chemical Reaction. Open Journal of Fluid Dynamics, 5, 364-379. http://dx.doi.org/10.4236/ojfd.2015.54036 
owing to its general occurrence in various fields of engineering and science. Especially, the subject of mass and heat transfer with heat radiation, chemical reaction has significant role in hydrometallurgical and chemical industries. For a moving plate, a chemical reaction takes place in legion chemical processes between a fluid and foreign mass. This sue is involved in many industrial usages such as glassware or ceramics manufacturing, food processing and production of polymers. The convection study with mass and heat transfer plays a major role in the dispersion and formation of fog, design of chemical processing equipment's, temperature distribution, and moisture over agricultural fields and in the paper drying process.

Ahmed et al. [1] have identified the analysis for MHD rotating heat or mass transport phenomenon bounded by a vertical oscillating surface in the Mein of Darcian porous regime by using Numerical/Laplace transform. Characteristics of the heat and mass transfer in the Mien of chemical reaction and thermal radiation for a Newtonian incompressible fluid across an extending vertical surface having temperature dependent viscosity was studied by Kandasamy et al. [2]. Makinde [3] examined the free transient convection interaction of an absorbing, emitting plate with thermal radiation. Mukhopadhyay [4] performed an investigation on the results of heat transfer and thermal radiation on a mixed unsteady convective flow across an extending porous surface in porous medium.

Heat transfer analysis of a forced convective flow of the fluid past an embedded plate in a porous medium for an incompressible fluid was examined by Mukhopadhyay and Layek [5]. Muthucumaraswamy and Ganesan [6] looked at the impulsively started transient radiation-convection flow with vertical temperature consequences. An analysis of the chemical reaction, theoretically a result, with variable temperature on a vertical oscillating plate was given by Muthucumaraswamy [7]. Reddy et al. [8] investigated the consequences of unsteady natural MHD convective flow in a porous medium with constant mass diffusion and Newtonian heating. The effects of MHD radiating and chemically reacting fluid past a non-isothermal impulsively started vertical surface adjacent to a porous regime by using numerical analysis was discussed by Sahin Ahmed [9].

The importance of the present flow problem is to analyze the effects of thermal radiation and radiation absorption on the flow past an impulsively started infinite vertical plate with Newtonian heating and chemical reaction.

\section{Mathematical Analysis}

Free convective unsteady flow of the fluid for a vertical transfinite plate with Newtonian heating, past an impulsively started incompressible viscous fluid in the Mien of radiation and radiation absorption is studied. Along the plate and in the vertical upward direction, axis $\bar{x}^{*}$ is chosen and normal to the plate, axis $\bar{y}^{*}$ is considered. Initially the fluid and the plate are having same temperature $\bar{T}_{\infty}^{*}$ and the concentration $\bar{C}_{\infty}^{*}$ at all points in a stationary state for time $\bar{t}^{*} \leq 0$. The coordinate system and the flow model are shown in Figure 1. The plate is fixed with a velocity $u_{0}$ in the vertical direction into impulsive motion versus the gravitational field at time $\bar{t}^{*}>0$. We assumed that i) heat transfer rate and the local surface temperature $\bar{T}^{*}$ are proportional to one another from the surface, and near the plate concentration rises to $\bar{C}_{w}^{*}$ and ii) the consequences of viscous dissipation are negligible in the energy equation. Among the fluid and diffusing species, there is a first order chemical reaction. Since all the physical quantities are expressed in terms of $\bar{y}^{*}, \bar{t}^{*}$ only and are free from $\bar{x}^{*}$ and in the direction of $\bar{x}^{*}$, the plate is considered transfinite.

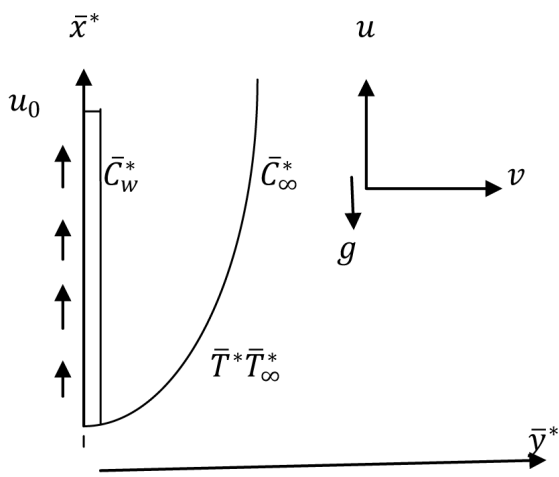

Figure 1. A sketch of flowmodel and coordinate system. 
The equations for this present flow, by the Boussinesq estimation are as follows

$$
\begin{gathered}
\frac{\partial \bar{u}^{*}}{\partial \bar{t}^{*}}=g \beta\left(\bar{T}^{*}-\bar{T}_{\infty}^{*}\right)+g \bar{\beta}\left(\bar{C}^{*}-\bar{C}_{\infty}^{*}\right)+v \frac{\partial^{2} \bar{u}^{*}}{\partial \bar{y}^{* 2}} \\
\rho C_{p} \frac{\partial \bar{T}^{*}}{\partial \bar{t}^{*}}=\kappa \frac{\partial^{2} \bar{T}^{*}}{\partial \bar{y}^{*^{2}}}+\bar{Q}_{1}^{*}\left(\bar{C}^{*}-\bar{C}_{\infty}^{*}\right)-\frac{\partial \bar{q}_{r}^{*}}{\partial \bar{y}^{*}} \\
\frac{\partial \bar{C}^{*}}{\partial \bar{t}^{*}}=\mathcal{D} \frac{\partial^{2} \bar{C}^{*}}{\partial y^{* 2}}-K_{r}\left(\bar{C}^{*}-\bar{C}_{\infty}^{*}\right)
\end{gathered}
$$

and the connected conditions for this flow are

$$
\left.\begin{array}{c}
\text { at } \bar{t}^{*} \leq 0: \bar{u}^{*}=0, \bar{T}^{*}=\bar{T}_{\infty}^{*}, \bar{C}^{*}=\bar{C}_{\infty}^{*} \forall \bar{y}^{*} \\
\text { at } \bar{t}^{*}>0: \bar{u}^{*}=u_{0}, \frac{\partial \bar{T}^{*}}{\partial \bar{y}^{*}}=-\frac{h}{\kappa} T^{*}, \bar{C}^{*}=\bar{C}_{w}^{*} \text { for } \bar{y}^{*}=0 \\
\bar{u}^{*}=0, \bar{T}^{*} \rightarrow \bar{T}_{\infty}^{*}, \bar{C}^{*} \rightarrow \bar{C}_{\infty}^{*} \text { as } \bar{y}^{*} \rightarrow \infty
\end{array}\right\}
$$

Here $A=\frac{u_{0}^{2}}{v}$

The term of radiative heat flux by the Rosseland estimation is given by

$$
\bar{q}_{r}^{*}=-\frac{4 \sigma_{s}}{3 k_{c}} \frac{\partial \bar{T}^{* 4}}{\partial \bar{y}^{*}}
$$

But here with in the flow, presuming that the deviation in temperatures can be showed as a linear combination of the temperatures and around $\bar{T}_{\infty}^{*}$, which is found by expanding $\bar{T}^{* 4}$ in a Taylor's series as follows:

$$
\bar{T}^{* 4}=\bar{T}_{\infty}^{* 4}+4 \bar{T}_{\infty}^{* 3}\left(\bar{T}^{*}-\bar{T}_{\infty}^{*}\right)+6 \bar{T}_{\infty}^{* 2}\left(\bar{T}^{*}-\bar{T}_{\infty}^{*}\right)+\cdots
$$

and ignoring the higher ordered terms, beyond the first degree, we get

$$
\bar{T}^{* 4} \cong 4 \bar{T}_{\infty}^{* 3} \bar{T}^{*}-3 \bar{T}_{\infty}^{* 4}
$$

Differentiating Equation (5) with respect to $\bar{y}^{*}$ and applying Equation (6), we get

$$
\frac{\partial \bar{q}_{r}^{*}}{\partial \bar{y}^{*}}=-\frac{16 \sigma_{s} \bar{T}_{\infty}^{* 3}}{3 k_{c}} \frac{\partial^{2} \bar{T}^{*}}{\partial \bar{y}^{*^{2}}}
$$

We have inserted the non-dimensional succeeding measures

$$
\left.\begin{array}{l}
U=\frac{\bar{u}^{*}}{u_{0}}, t=\frac{\bar{t}^{*} u_{0}^{2}}{v}, y=\frac{\bar{y}^{*} u_{0}}{v}, \operatorname{Pr}=\frac{\mu c_{p}}{\kappa}, G r=\frac{g \beta v \bar{T}_{\infty}^{*}}{u_{0}^{3}}, \\
\theta=\frac{\bar{T}^{*}-\bar{T}_{\infty}^{*}}{\bar{T}_{\infty}^{*}}, S c=\frac{v}{\mathcal{D}}, Q_{1}=\frac{v \bar{Q}_{1}^{*}\left(\bar{C}_{w}^{*}-\bar{C}_{\infty}^{*}\right)}{\rho C_{p} u_{0}^{2} \bar{T}_{\infty}^{*}}, G m=\frac{g \bar{\beta} v\left(\bar{C}_{w}^{*}-\bar{C}_{\infty}^{*}\right)}{u_{0}^{3}}, \\
C=\frac{\bar{C}^{*}-\bar{C}_{\infty}^{*}}{\bar{C}_{w}^{*}-\bar{C}_{\infty}^{*}}, K=\frac{K_{r} v}{u_{0}^{2}}, N_{r}=\frac{16 \sigma_{s} \bar{T}_{\infty}^{* 3}}{3 \kappa k_{c}}
\end{array}\right\}
$$

The Equations (1)-(3) are reduced into the following forms by using the Equations (8) and (9) as follows

$$
\frac{\partial U}{\partial t}=G_{r} \theta+G_{m} C+\frac{\partial^{2} u}{\partial y^{2}}
$$




$$
\begin{gathered}
\frac{\partial \theta}{\partial t}=\left(\frac{1+N_{r}}{P_{r}}\right) \frac{\partial^{2} \theta}{\partial y^{2}}+Q_{1} C \\
\frac{\partial C}{\partial t}=\frac{1}{S_{c}} \frac{\partial^{2} C}{\partial y^{2}}-K C
\end{gathered}
$$

In non-dimensional form, the conditions reduce to as follows

$$
\left.\begin{array}{rl}
\text { at } t \leq 0: U & =0, \theta=0, C=0 \quad \forall y \\
\text { at } t>0: U & =1, \frac{\partial \theta}{\partial y}=-(1+\theta), C=1 \text { for } y=0 \\
U & =0, \theta \rightarrow 0, C \rightarrow 0 \quad \text { as } y \rightarrow \infty
\end{array}\right\}
$$

\section{Solution of the Problem}

The non-dimensional Equations (10)-(12) associated with the conditions given by Equations (13) are evolved by the method of Laplace transforms, and therefore the results of concentration, temperature and velocity are given by

$$
\begin{aligned}
& C(y, t)=B_{6} \\
& \theta(y, t)=-\frac{1}{\sqrt{a}} B_{1}-B_{2}+\frac{1}{\sqrt{a}} B_{3}+N_{1}\left(M_{1} B_{1}+M_{2} B_{2}+M_{3} B_{4}+M_{4} B_{5}+M_{5} B_{3}\right)+N_{2}\left(B_{6}-B_{7}\right) \\
& U(y, t)=A_{1}+C_{1}\left(A_{4}+A_{3}-A_{5}-A_{27}+A_{2}-A_{28}\right) \\
& +C_{2}\left(A_{12}+A_{13}+A_{6}+A_{11}+A_{7}+A_{10}+A_{14}+A_{8}+A_{15}+A_{9}\right) \\
& +C_{3}\left[M_{6}\left(m_{1}-m_{2}\right)+M_{7}\left(A_{1}-A_{12}\right)+M_{8}\left(A_{16}-A_{22}\right)\right. \\
& +M_{9}\left(A_{17}-A_{23}\right)+M_{10}\left(A_{18}-A_{24}\right)+M_{11}\left(A_{19}-A_{25}\right) \\
& \left.+M_{12}\left(A_{20}-A_{26}\right)\right]+C_{4}\left[A_{1}-A_{29}-A_{30}+A_{31}\right]
\end{aligned}
$$

\section{The Rate of Heat Transfer}

In dimensionless form, heat transfer rate from the temperature gradient is

$$
N u=-\left(\frac{\partial \theta}{\partial y}\right)_{y=0}
$$

From Equations (15) and (17), we get

$$
\begin{aligned}
N u= & \exp (a t) \operatorname{erfc}(-\sqrt{a t})+N_{1}\left[\frac{M_{2}}{\sqrt{\pi a t}}-M_{3} \frac{b}{\sqrt{a}} \exp (-b t) \operatorname{erfc}(i \sqrt{b t})\right. \\
& +\frac{i \sqrt{b}}{\sqrt{\pi a t}} \mathrm{e}^{-2 b t}\left(M_{4}-M_{3}\right)-M_{4} \frac{b}{\sqrt{a}} \exp (-b t) \operatorname{erfc}(-i \sqrt{b t}) \\
& \left.+M_{5} \sqrt{a} \exp (a t) \operatorname{erfc}(-\sqrt{a t})+M_{5} \frac{1}{\sqrt{\pi t}}\right]+N_{2} \sqrt{K S_{c}} \operatorname{erf}(\sqrt{K t}) \\
& -N_{2} \exp (-b t) \sqrt{S_{c}(K-b)} \operatorname{erf}(\sqrt{(K-b) t})
\end{aligned}
$$

\section{The Rate of Mass Transfer}

In dimensionless form, mass transfer rate from the concentration gradient is 
S. Ravi et al.

$$
S h=-\left(\frac{\partial c}{\partial y}\right)_{y=0}
$$

From Equations (14) and (19), we get

$$
S h=\sqrt{K S_{c}} \operatorname{erf}(\sqrt{K t})+\sqrt{\frac{S_{c}}{\pi t}} \exp (-K t)
$$

6. Deduction

The effects of this analysis are in good agreement with the results given by Rajesh [8] in the absence of parameter radiation absorption $\left(Q_{1}\right)$ and radiation $\left(N_{r}\right)$, where

$$
\begin{aligned}
& B_{1}=\frac{1}{\sqrt{\pi t}} \exp \left(-\frac{y^{2}}{4 a t}\right), B_{2}=\operatorname{erfc}\left(\frac{y}{2 \sqrt{a t}}\right) A_{1}=\operatorname{erfc}\left(\frac{y}{2 \sqrt{t}}\right) \\
& B_{3}=\frac{1}{\sqrt{\pi t}} \exp \left(-\frac{y^{2}}{4 a t}\right)+\sqrt{a} \exp (-y+a t) \operatorname{erfc}\left(-\sqrt{a t}+\frac{y}{2 \sqrt{a t}}\right) \\
& B_{4}=\frac{1}{\sqrt{\pi t}} \exp \left(-\frac{y^{2}}{4 a t}\right)-i \sqrt{b} \exp \left(\frac{i y \sqrt{b}}{\sqrt{a}}-b t\right) \operatorname{erfc}\left(i \sqrt{b t}+\frac{y}{2 \sqrt{a t}}\right) \\
& B_{5}=\frac{1}{\sqrt{\pi t}} \exp \left(-\frac{y^{2}}{4 a t}\right)+i \sqrt{b} \exp \left(-\frac{i y \sqrt{b}}{\sqrt{a}}-b t\right) \operatorname{erfc}\left(-i \sqrt{b t}+\frac{y}{2 \sqrt{a t}}\right) \\
& B_{6}=\frac{1}{2}\left[\operatorname{erfc}\left(\frac{y \sqrt{S_{c}}}{2 \sqrt{t}}-\sqrt{K t}\right) \exp \left(-y \sqrt{K S_{c}}\right)+\operatorname{erfc}\left(\frac{y \sqrt{S_{c}}}{2 \sqrt{t}}+\sqrt{K t}\right) \exp \left(y \sqrt{K S_{c}}\right)\right] \\
& B_{7}=\frac{\exp (-b t)}{2}\left\{\operatorname{erfc}\left(\frac{y \sqrt{S_{c}}}{2 \sqrt{t}}-\sqrt{(K-b) t}\right) \exp \left(-y \sqrt{S_{c}(K-b)}\right)\right. \\
& \left.+\operatorname{erfc}\left(\frac{y \sqrt{S_{c}}}{2 \sqrt{t}}+\sqrt{(K-b) t}\right) \exp \left(y \sqrt{S_{c}(K-b)}\right)\right\} \\
& A_{2}=\frac{1}{b(b-d)}\left[\frac { \operatorname { e x p } ( - b t ) } { 2 } \left\{\operatorname{erfc}\left(\frac{y \sqrt{S_{c}}}{2 \sqrt{t}}-\sqrt{(K-b) t}\right) \exp \left(-y \sqrt{S_{c}(K-b)}\right)\right.\right. \\
& \left.\left.+\operatorname{erfc}\left(\frac{y \sqrt{S_{c}}}{2 \sqrt{t}}+\sqrt{(K-b) t}\right) \exp \left(y \sqrt{S_{c}(K-b)}\right)\right\}\right] \\
& A_{3}=\frac{1}{d(d-b)}\left[\frac { \operatorname { e x p } ( - d t ) } { 2 } \left\{\operatorname{erfc}\left(\frac{y \sqrt{S_{c}}}{2 \sqrt{t}}-\sqrt{(K-d) t}\right) \exp \left(-y \sqrt{S_{c}(K-d)}\right)\right.\right. \\
& \left.\left.+\operatorname{erfc}\left(\frac{y \sqrt{S_{c}}}{2 \sqrt{t}}+\sqrt{(K-d) t}\right) \exp \left(y \sqrt{S_{c}(K-d)}\right)\right\}\right]
\end{aligned}
$$

368 
S. Ravi et al.

$$
\begin{aligned}
& A_{4}=\frac{1}{b d}\left[\frac{1}{2}\left\{\operatorname{erfc}\left(\frac{y \sqrt{S_{c}}}{2 \sqrt{t}}-\sqrt{K t}\right) \exp \left(-y \sqrt{S_{c} K}\right)+\operatorname{erfc}\left(\frac{y \sqrt{S_{c}}}{2 \sqrt{t}}+\sqrt{K t}\right) \exp \left(y \sqrt{S_{c} K}\right)\right\}\right] \\
& A_{5}=\frac{1}{b(b-d)}\left[\frac{\exp (-b t)}{2} \operatorname{erfc}\left(\frac{y}{2 \sqrt{t}}-\sqrt{-b t}\right) \exp (-y \sqrt{-b})+\operatorname{erfc}\left(\frac{y}{2 \sqrt{t}}+\sqrt{-b t}\right) \exp (y \sqrt{-b})\right] \\
& A_{6}=-\frac{1}{\sqrt{\pi a t}} \exp \left(-\frac{y^{2}}{4 t}\right), \quad A_{7}=-A_{1}, \quad A_{8}=-\sqrt{a} A_{16}, \quad A_{9}=-a A_{17} \\
& A_{10}=\frac{1}{\sqrt{a}}\left[\frac{1}{\sqrt{\pi t}} \exp \left(-\frac{y^{2}}{4 t}\right)+\sqrt{a} \exp (-y \sqrt{a}+a t) \operatorname{erfc}\left(-\sqrt{a t}+\frac{y}{2 \sqrt{t}}\right)\right] \\
& A_{11}=\frac{1}{\sqrt{a}}\left[\frac{1}{\sqrt{\pi t}} \exp \left(-\frac{y^{2}}{4 a t}\right)\right], \quad A_{12}=\operatorname{erfc}\left(\frac{y}{2 \sqrt{a t}}\right), \quad A_{13}=\sqrt{a} A_{22}, \quad A_{14}=a A_{23} \text {, } \\
& A_{15}=\frac{-1}{\sqrt{a}}\left[\frac{1}{\sqrt{\pi t}} \exp \left(-\frac{y^{2}}{4 a t}\right)+\sqrt{a} \exp (-y+a t) \operatorname{erfc}\left(-\sqrt{a t}+\frac{y}{2 \sqrt{a t}}\right)\right] \\
& A_{16}=2 \sqrt{\frac{t}{\pi}} \exp \left(-\frac{y^{2}}{4 t}\right)-y \operatorname{erfc}\left(\frac{y}{2 \sqrt{t}}\right) \\
& A_{17}=\left(t+\frac{y^{2}}{2}\right) \operatorname{erfc}\left(\frac{y}{2 \sqrt{t}}\right)-y \sqrt{\frac{t}{\pi}} \exp \left(-\frac{y^{2}}{4 t}\right) \\
& A_{18}=\frac{1}{\sqrt{\pi t}} \exp \left(-\frac{y^{2}}{4 t}\right)-i \sqrt{b} \exp (i \sqrt{b} y-b t) \operatorname{erfc}\left(i \sqrt{b t}+\frac{y}{2 \sqrt{t}}\right) \\
& A_{19}=\frac{1}{\sqrt{\pi t}} \exp \left(-\frac{y^{2}}{4 t}\right)+i \sqrt{b} \exp (-i \sqrt{b} y-b t) \operatorname{erfc}\left(-i \sqrt{b t}+\frac{y}{2 \sqrt{t}}\right) \\
& A_{20}=\frac{1}{\sqrt{\pi t}} \exp \left(-\frac{y^{2}}{4 t}\right)+\sqrt{a} \exp (-\sqrt{a} y+a t) \operatorname{erfc}\left(-\sqrt{a t}+\frac{y}{2 \sqrt{t}}\right), \\
& A_{22}=2 \sqrt{\frac{t}{\pi}} \exp \left(-\frac{y^{2}}{4 a t}\right)-\frac{y}{\sqrt{a}} \operatorname{erfc}\left(\frac{y}{2 \sqrt{a t}}\right) A_{28}=\frac{1}{b d} A_{1} \\
& A_{23}=\left(t+\frac{y^{2}}{2 a}\right) \operatorname{erfc}\left(\frac{y}{2 \sqrt{a t}}\right)-y \sqrt{\frac{t}{a \pi}} \exp \left(-\frac{y^{2}}{4 a t}\right) \\
& A_{24}=\frac{1}{\sqrt{\pi t}} \exp \left(-\frac{y^{2}}{4 a t}\right)-i \sqrt{b} \exp \left(i \sqrt{b} \frac{y}{\sqrt{a}}-b t\right) \operatorname{erfc}\left(i \sqrt{b t}+\frac{y}{2 \sqrt{a t}}\right) \\
& A_{25}=\frac{1}{\sqrt{\pi t}} \exp \left(-\frac{y^{2}}{4 a t}\right)+i \sqrt{b} \exp \left(-i \sqrt{b} \frac{y}{\sqrt{a}}-b t\right) \operatorname{erfc}\left(-i \sqrt{b t}+\frac{y}{2 \sqrt{a t}}\right)
\end{aligned}
$$

369 


$$
\begin{gathered}
A_{26}=\frac{1}{\sqrt{\pi t}} \exp \left(-\frac{y^{2}}{4 a t}\right)+\sqrt{a} \exp (-y+a t) \operatorname{erfc}\left(-\sqrt{a t}+\frac{y}{2 \sqrt{a t}}\right) \\
A_{27}=\frac{1}{d(d-b)}\left[\frac{\exp (-d t)}{2} \operatorname{erfc}\left(\frac{y}{2 \sqrt{t}}-\sqrt{-d t}\right) \exp (-y \sqrt{-d})+\operatorname{erfc}\left(\frac{y}{2 \sqrt{t}}+\sqrt{-d t}\right) \exp (y \sqrt{-d})\right] \\
A_{29}=\frac{\exp (-d t)}{2}\left\{\operatorname{erfc}\left(\frac{y}{2 \sqrt{t}}-\sqrt{-d t}\right) \exp (-y \sqrt{-d})+\operatorname{erfc}\left(\frac{y}{2 \sqrt{t}}+\sqrt{-d t}\right) \exp (y \sqrt{-d})\right\} \\
A_{30}=\frac{1}{2}\left[\operatorname{erfc}\left(\frac{y \sqrt{S_{c}}}{2 \sqrt{t}}-\sqrt{K t}\right) \exp \left(-y \sqrt{K S_{c}}\right)+\operatorname{erfc}\left(\frac{y \sqrt{S_{c}}}{2 \sqrt{t}}+\sqrt{K t}\right) \exp \left(y \sqrt{K S_{c}}\right)\right] \\
A_{31}=\frac{\exp (-d t)}{2}\left\{\operatorname{erfc}\left(\frac{y \sqrt{S_{c}}}{2 \sqrt{t}}-\sqrt{(K-d) t}\right) \exp \left(-y \sqrt{S_{c}(K-d)}\right)\right. \\
\left.+\operatorname{erfc}\left(\frac{y \sqrt{S_{c}}}{2 \sqrt{t}}+\sqrt{(K-d) t}\right) \exp \left(y \sqrt{S_{c}(K-d)}\right)\right\}
\end{gathered}
$$

\section{Results and Discussion}

In this field of study, in order to examine the consequences of velocity field, temperature profile and concentration profiles by allotting numerical values for several arguments for both water vapor and ammonia, Prandtl number $(P r)$ and Schmidt number $(S c)$ values are considered.

For several values of different arguments the velocities are analyzed and are presented in Figures 2-7 at time $t$ $=0.4$ respectively, for both the types of heating $(G r<0, G m<0)$ plate and cooling $(G r>0, G m>0)$ plate. Figure 2 depicts the result of Schmidt number $(S c)$ at time $t=0.4$ on the flow. With an increase in Sc, it is noticed that the velocity increases for heating of the plate and decreases for cooling of the plate and as $S c$ increases at $t=$ 0.4 from $0.22-0.30$ and to 0.60 , the maximum velocity of the fluid decreases by $5.7 \%-10 \%$ for ammonia and by $5.6 \%-10.9 \%$ for water vapor in the case of cooling plate and the minimum velocity of the fluid increases by $10 \%$ and $16.58 \%$ for ammonia and by $10.26 \%$ - $17 \%$ for water vapor in the case of heating plate. Prandtl number $(P r)$ effects on the flow are expressed in Figure 4 at time $t=0.4$. It is identified that there is an increase in velocity near the plate and then decreases with a point of separation moving far away from the plate in the type of

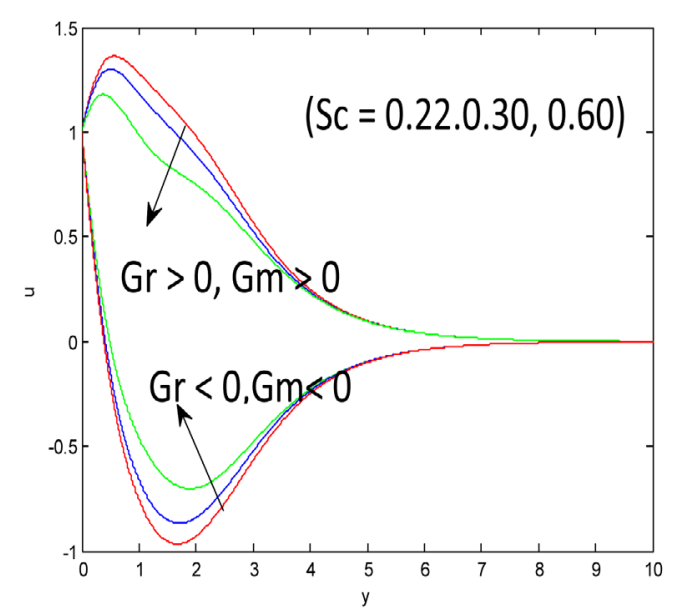

Figure 2. Velocity profile shows the effect of $S c$ and $\operatorname{Pr}=0.71, K=0.2, N r=0.1, Q_{1}=0.1, t=0.4$. 


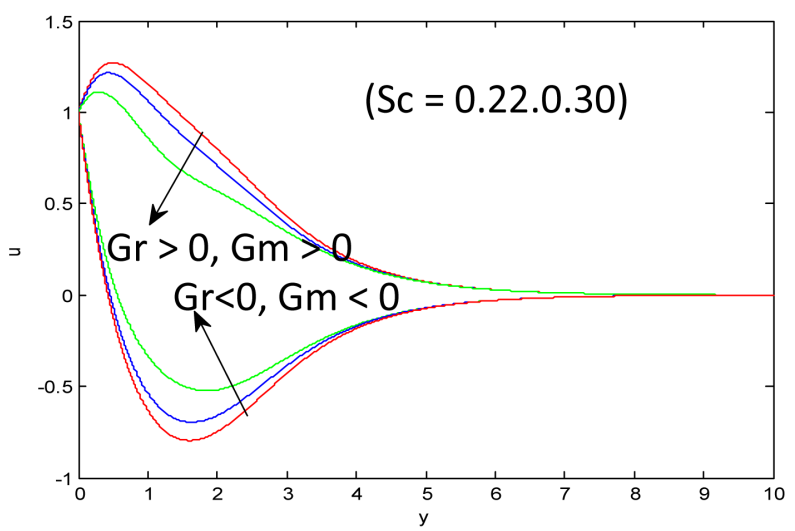

Figure 3. Velocity profile displays the result of $S c$ and $\mathrm{Pr}=$ $0.71, K=0.2, N r=0, Q_{1}=0, t=0.4$.

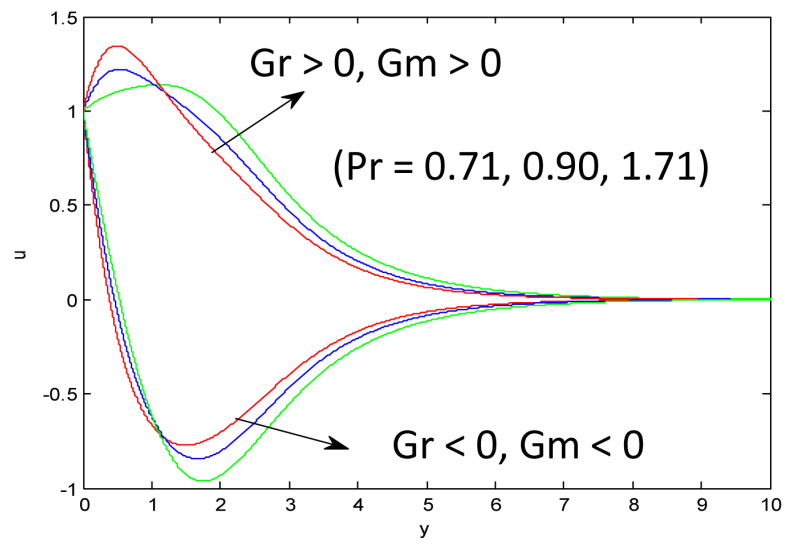

Figure 4. Velocity profile shows the effect of $\mathrm{Pr}$ and $\mathrm{Sc}=$ $0.22, K=0.2, N r=0.1, Q_{1}=0.1, t=0.4$.

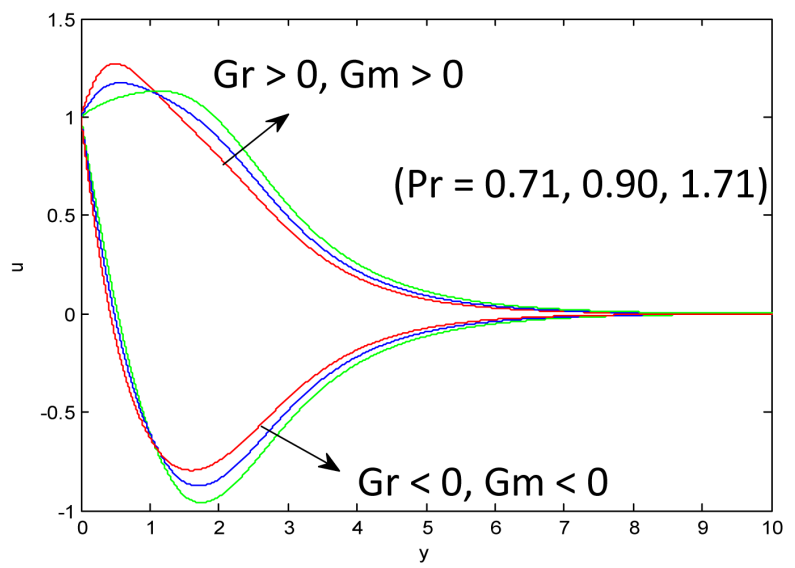

Figure 5. Velocity profile displays the result of $\mathrm{Pr}$ and $\mathrm{Sc}=$ $0.22, K=0.2, N r=0, Q_{1}=0, t=0.4$.

heating and in the instance of cooling plate, the reverse effect is found with the increase of Pr. Owing to the variation in parameter of chemical reaction $(K)$, Figure 6 reveals the consequence of velocity profiles at time $t=$ 0.4. As $K$ increases, it is found that, there is an increase velocity in the plate of heating type and the velocity decreases in the plate of cooling type and as $K$ increases at $t=0.4$ from $0.2-2$ and to 5 , the maximum velocity of 


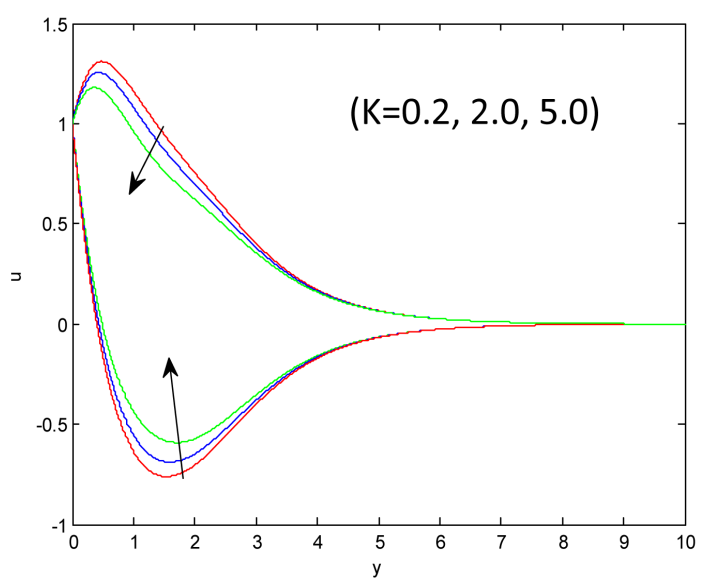

Figure 6. Velocity profile shows the effect of $K$ and $S c$ $=0.22, \operatorname{Pr}=0.71, \mathrm{Nr}=0.1, Q_{1}=0.1, t=0.4$.

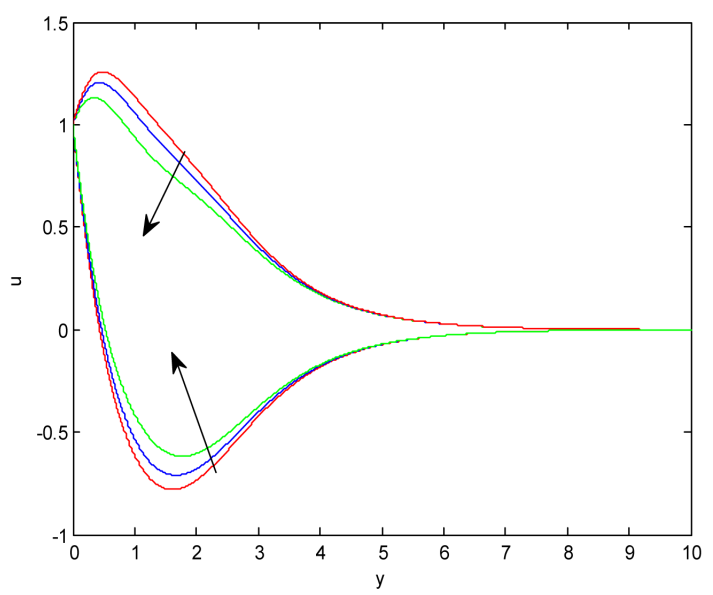

Figure 7. Velocity profile displays the result of $K$ and $S c=0.22, \operatorname{Pr}=0.71, N r=0, Q_{1}=0, t=0.4$.

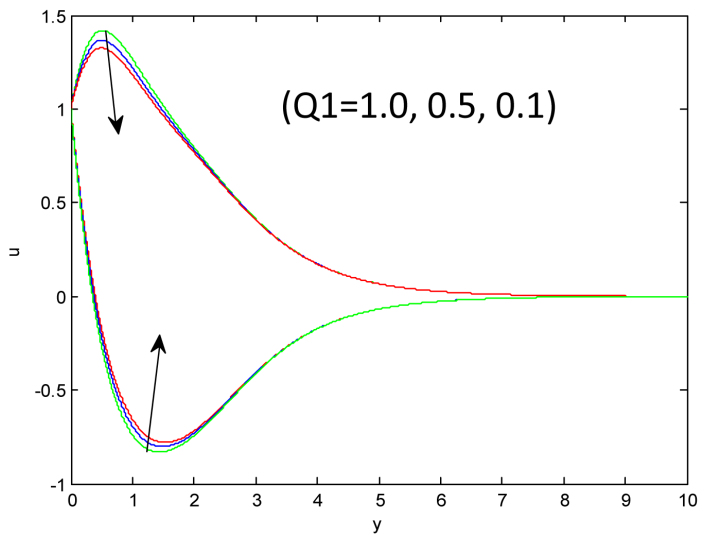

Figure 8. Velocity profile shows the effect of $K$ and $S c$ $=0.22, \operatorname{Pr}=0.71, N r=0.1, Q_{1}=0.1, t=0.4$.

the fluid decreases by $7.4 \%-6.8 \%$ for ammonia and by $7.1 \%-7.4 \%$ for water vapor in the case of cooling plate and the minimum velocity of the fluid increases by $8.4 \%-9.04 \%$ for ammonia and by $5.56 \%-9.61 \%$ for water vapor in the case of heating plate. 
For various values of $N r$ (radiation parameter), the velocity profile is shown in Figure 8 at $t=0.4$. It is seen that there is a decrease in velocity near the plate and then increases with a point of separation moving away from the plate in the cooling case and the phenomenon is reversed in the case of heating type with the decrease of $\mathrm{Nr}$. Figure 9 describes the effects of $Q_{1}$ (radiation absorption parameter) at $t=0.4$. As $Q_{1}$ decreases, it is observed that the velocity increases for heating plate and decreases for cooling plate. Figure 10 reveals the result of velocity profile at several times $(t=0.4,0.6,0.8)$. It is identified that there is a considerable decrease in velocity in the heating type and increase when the plate is cooled as time $(t)$ increases. The same results are noticed in the absence of thermal radiation and radiation absorption for different values of $S c, \operatorname{Pr}, K, t$ which are shown in Figure 3, Figure 5, Figure 7, and Figure 11. Hence, these results are in good agreement with the results of Rajesh [10].

The effect of temperature profile for several values of various parameters are studied and shown in Figures 12-17 at time $t=0.2$. It is observed from Figures 12-14 that the temperature rises with the fall in $S c$ and $K$. In Figures 15-17, it is observed that the temperature rises with the increase of $N r, Q_{1}$, and $t$. Moreover Figure 13 shows the results of velocity for different values of $\mathrm{Pr}$. It is noticed from the values that, velocity increases near the

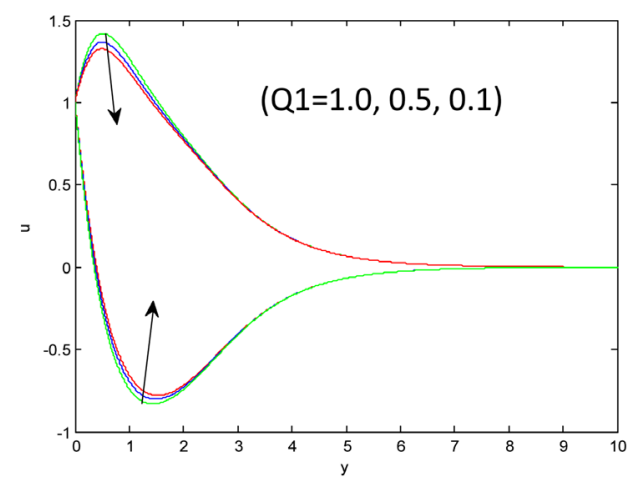

Figure 9. Velocity profile displays the result of $K$ and $S c=0.22, \operatorname{Pr}=0.71, N r=0, Q 1=0, t=0.4$.

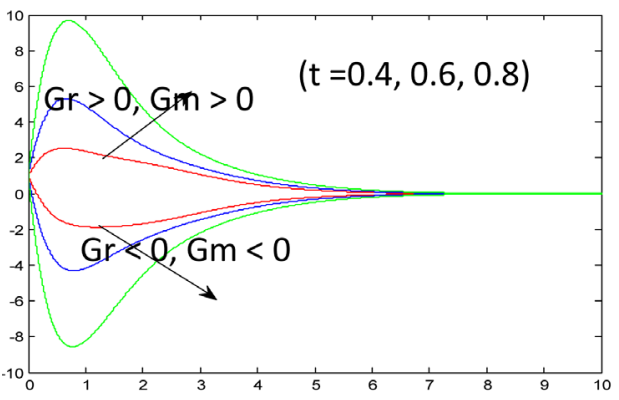

Figure 10. The effect of velocity profile fortand $\mathrm{Pr}=$ $0.71, K=0.2, N r=0.1, S c=0.22, Q_{1}=0.1$.

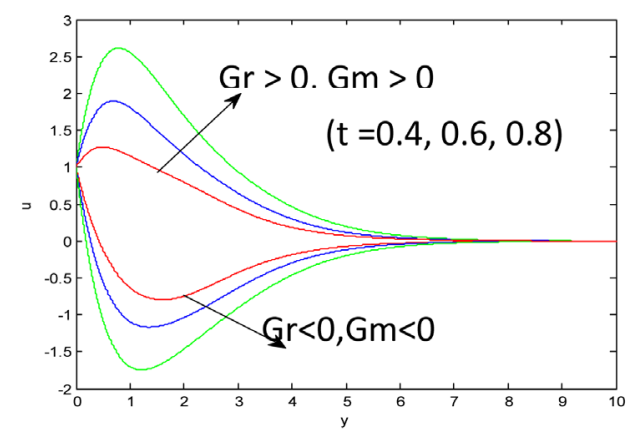

Figure 11. The result of velocity profile for tand $S c=$ $0.22, K=0.2, N r=0.1, Q_{1}=0.1, P r=0.71$. 


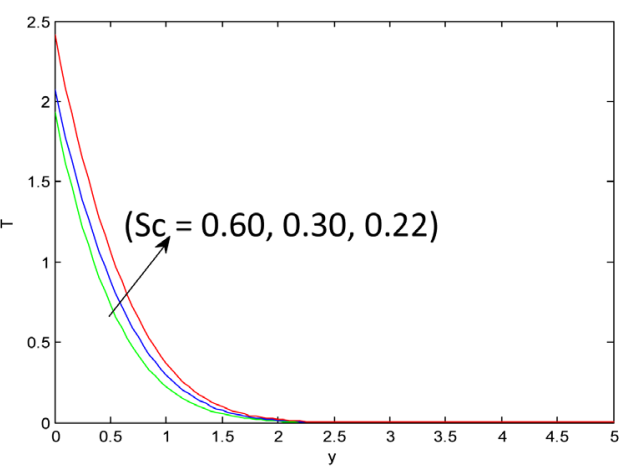

Figure 12. Temperature profile presents the effect of $S c$ and $\operatorname{Pr}=0.71, K=0.2, N r=0.1, Q_{1}=0.1, t=0.2$.

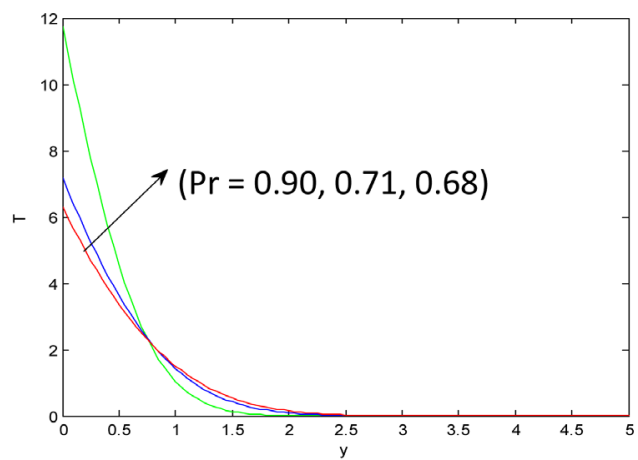

Figure 13. Temperature profile shows the resultof $\mathrm{Pr}$ and $S c=0.22, K=0.2, N r=0.1, Q_{1}=0.1, t=0.2$.

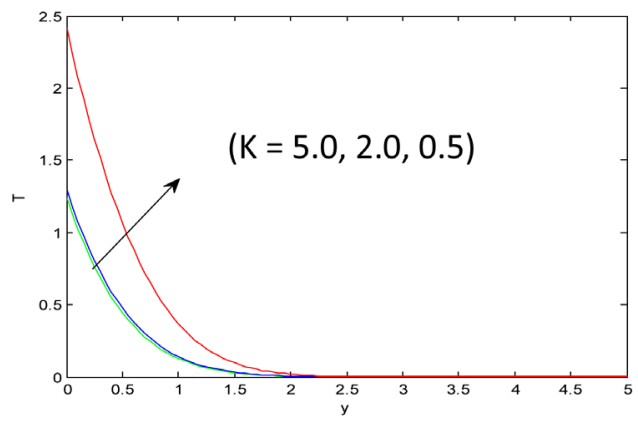

Figure 14. Temperature profile displays ensue of $K$ and $S c=0.22, \operatorname{Pr}=0.71, \mathrm{Nr}=0.1, Q_{1}=0.1, t=0.2$.

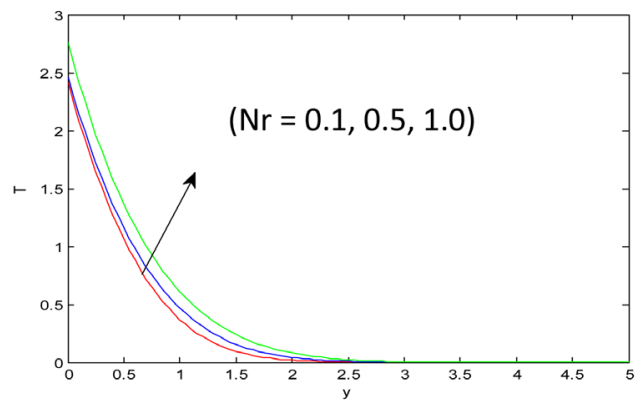

Figure 15. Temperature profile indicates the effect of $N r$ and $0.22, K=0.2, Q_{1}=0.1, P r=0.71, t=0.2$. 


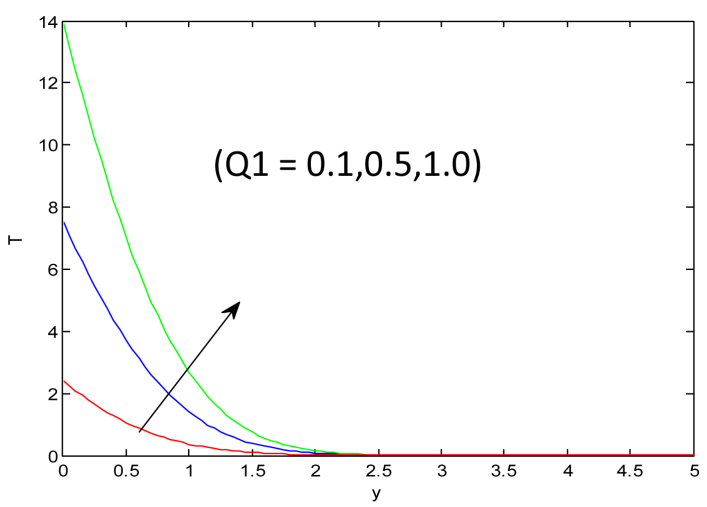

Figure 16. The effect of temperature profile for $Q_{1}$ and $\operatorname{Pr}=0.71, S c=0.22, N r=0.1, K=0.2, t=0.2$.

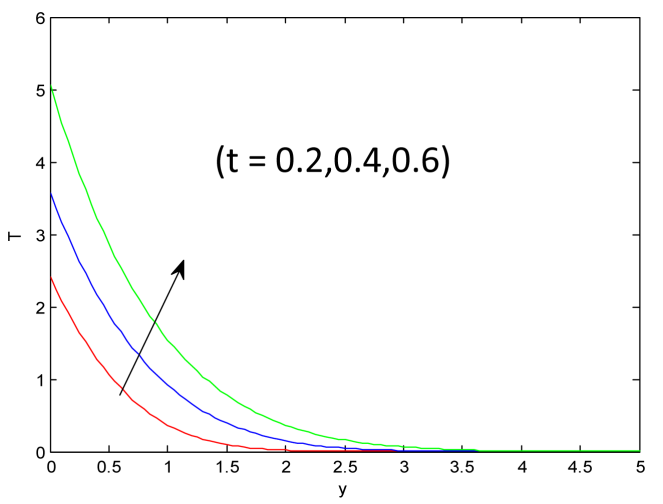

Figure 17. Theresult of temperature profile for tand $S c$ $=0.22, K=0.2, Q_{1}=0.1, P r=0.1, N r=0.1$.

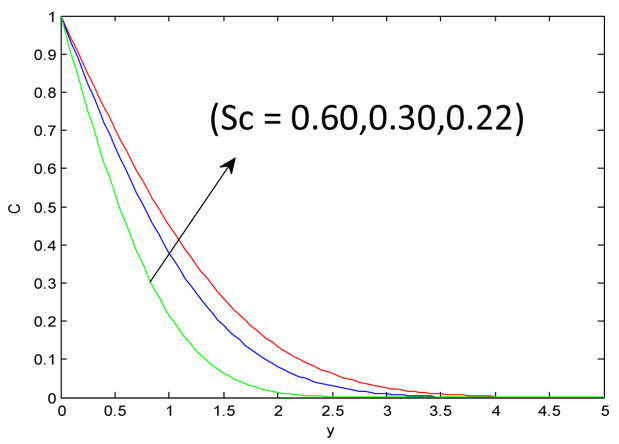

Figure 18. Concentration profile displays the effect of Sc and $K=0.2, t=0.2$.

plate and falls far away from the plate with a point of separation.

The effect of concentration profile for several values of various parameters is studied and is presented in Figures 18-20 at times 0.4. From Figure 18 and Figure 19, it is identified that with the decrease in $S c$ and $K$, the concentration increases. And from Figure 20, it is found that there is a rise in concentration with the rise in time $t$. For several values of various arguments, the Sherwood number versus time is presented in Figure 21 and Figure 22. It is identified that, there is an increase in Sherwood number for both hydrogen and water vapor with the increase of $S c$ and $K$.

For different values of various arguments for both hydrogen and water vapor, the Nusselt number versus time is shown in Figure 23. From this figure, it is observed that, there is a rise in Nusselt number with the fall in $\operatorname{Pr}$. 


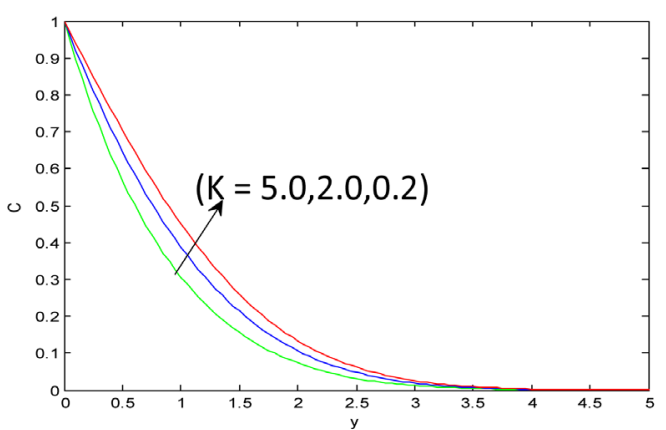

Figure 19. Concentration profile shows theresult of $K$ and $S c=0.22, t=0.2$.

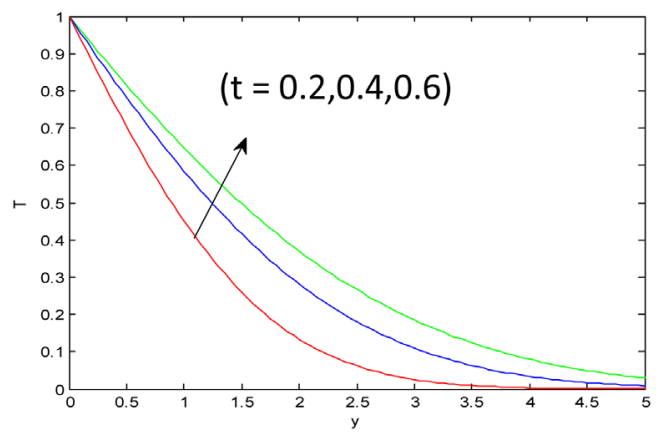

Figure 20. Concentration profile presents theensue of $t$ and $S_{c}=0.22, K=0.2$.

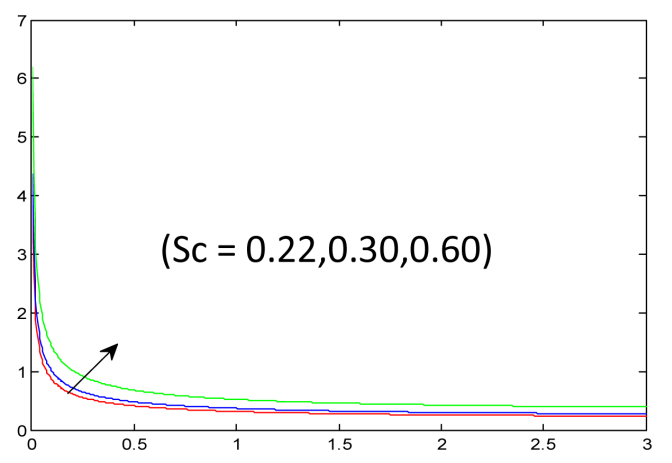

Figure 21. Effect of $S c$ on the real part of Sherwood number and $K=0.2$.

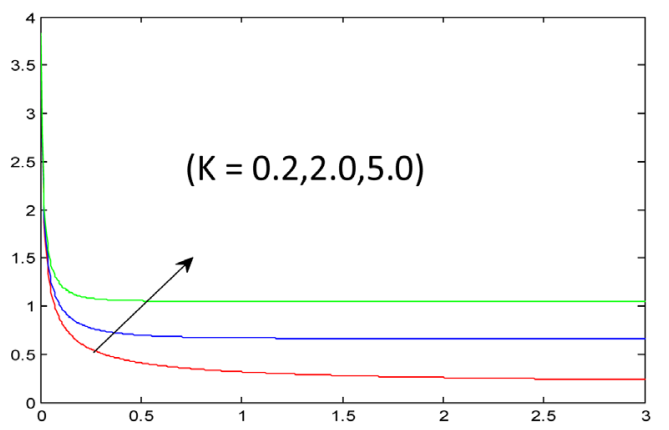

Figure 22. Result of $K$ on the real part of Sherwood number and $S_{c}=0.22$. 


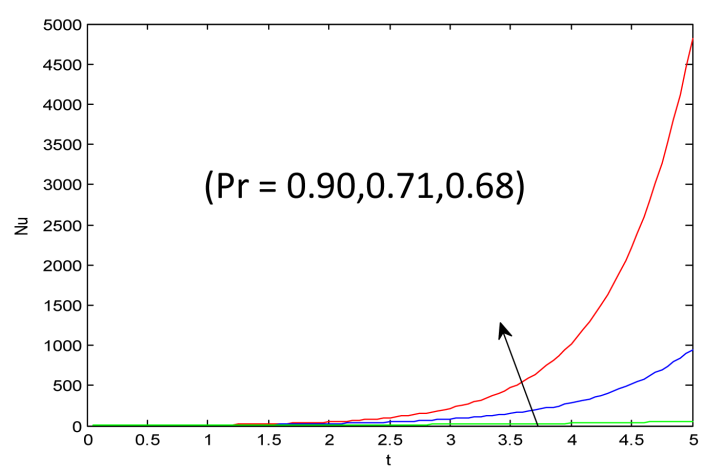

Figure 23. Effect of $\mathrm{Pr}$ on the real part of nusselt number and $K=0.2, S c=0.22, N r=0.1, Q_{1}=0.1$.

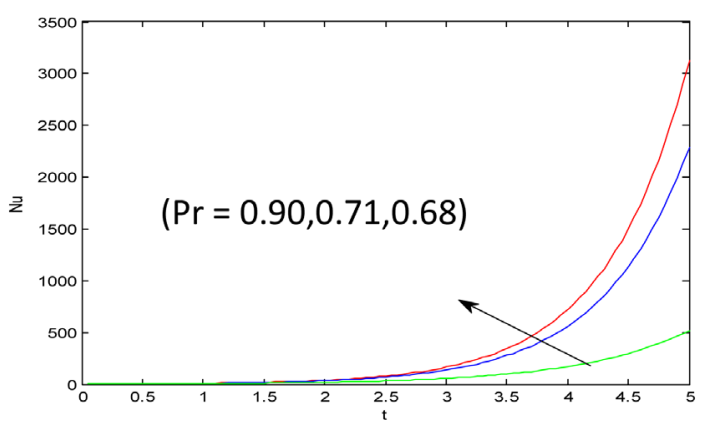

Figure 24. Result of $\mathrm{Pr}$ on the real part of nusselt number and $K=0.2, S c=0.22, N r=0, Q_{1}=0$.

And the same results are noticed in the absence of thermal radiation and radiation absorption for different values of $\operatorname{Pr}$ which is shown in Figure 24. Hence, these results are in good agreement with the results of Rajesh [10].

\section{References}

[1] Ahmed, S., Batin, A. and Chamka, A.J. (2015) Numerical/Laplace Transform Analysis for MHD Rotating Heat/ Mass Transport in a Darcian Porous Regime Bounded by an Oscillating Vertical Surface. Alexandria Engineering Journal, 54, 45-54. http://dx.doi.org/10.1016/j.aej.2014.11.006

[2] Kandasamy, R., Muhaimin, I. and Saim, H.B. (2010) Group Analysis for the Effects of the Temperature Dependent Fluid Viscosity and Chemical Reaction on Free Convective Heat and Mass Transfer. Journal of Applied Mechanics and Technical Physics, 51, 887-897. http://dx.doi.org/10.1007/s10808-010-0110-2

[3] Makinde, O.D. (2005) Free Convection Flow with Thermal Radiation and Mass Transfer Past a Moving Vertical Porous Plate. International Communications in Heat and Mass Transfer, 32, 1411-1419. http://dx.doi.org/10.1016/j.icheatmasstransfer.2005.07.005

[4] Mukhopadhyay, S. (2009) Effect of Thermal Radiation on Unsteady Mixed Convection Flow and Heat Transfer over a Porous Stretching Surface in Porous Medium. International Journal of Heat and Mass Transfer, 52, 3261-3265. http://dx.doi.org/10.1016/j.ijheatmasstransfer.2008.12.029

[5] Mukhopadhyay, S. and Layek, G.C. (2009) Radiation Effect on Forced Convective Flow and Heat Transfer over a Porous Plate in a Porous Medium. Meccanica, 44, 587-597. http://dx.doi.org/10.1007/s11012-009-9211-5

[6] Muthucumaraswamy, R. and Ganesan, P. (2003) Radiation Effects on Flow Past an Impulsively Started Infinite Vertical Plate with Variable Temperature. International Journal of Applied Mechanics and Engineering, 8, 125-129.

[7] Muthucumaraswamy, R. (2010) Chemical Reaction Effects on Vertical Oscillating Plate with Variable Temperature. Chemical Industry \& Chemical Engineering Quarterly, 16, 167-173.

[8] Hussanan, A., Ismail, Z., Khan, I., Hussein, A.G. and Shafie, S. (2014) Unsteady Boundary Layer MHD Free Convection Flow in a Porous Medium with Constant Mass Diffusion and Newtonian Heating. The European Physical Journal Plus, 129, 46.

[9] Ahmed, S. (2014) Numerical Analysis for MHD Chemically Reacting and Radiating Fluid Past a Non Isothermal Im- 
pulsively Started Vertical Surface Adjacent to a Porous Regime. Ain Shams Engineering Journal, 5, 923-933. http://dx.doi.org/10.1016/j.asej.2014.02.005

[10] Rajesh, V. (2012) Effects of Mass Transfer on Flow Past an Impulsively Started Infinite Vertical Plate with Newtonian Heating and Chemical Reaction. Journal of Engineering Physics and Thermophysics, 85, 221-228.

http://dx.doi.org/10.1007/s10891-012-0642-9

\section{Nomenclature}

$\bar{C}_{\infty}^{*}$ : concentration far away from the plate;

$\bar{C}^{*}$ : concentration;

$C$ : non-dimensional species concentration;

$\bar{C}_{w}^{*}$ : concentration at the plate;

$D$ : molecular diffusivity;

$C_{p}$ : specific heat at constant pressure;

$G_{r}$ : thermal grashoff number;

$G_{m}$ : mass grashoff number;

$h$ : coefficient of heat transfer;

$g$ : acceleration due to gravity;

$K_{r}$ : parameter of chemical reaction;

$K$ : non-dimensional parameter of chemical reaction;

$q_{r}$ : radiative heat flux;

$Q_{1}$ : parameter of radiation absorption;

$k_{c}$ : mean absorption coefficient;

$\sigma_{s}$ : Stefan-Boltzmann constant;

$N_{r}$ : parameter of radiation;

$\operatorname{Pr}$ : Prandtl number;

Sc : Schmidt number;

Sh: Sherwood number;

$\mathrm{Nu}$ : Nusselt number;

$\bar{T}^{*}$ : temperature;

$\bar{T}_{\infty}^{*}$, ambient temperature;

$t$ : non-dimensional time;

$\bar{t}^{*}:$ time;

$U$ : non-dimensional velocity of the in the $\bar{x}^{*}$, direction;

$\bar{u}^{*}$ : velocity of the fluid;

$u_{0}$ : plate velocity;

$\bar{x}^{*}$ and $\bar{y}^{*}$ : Cartesian coordinates along the plate and normal to the plate;

$y$ : non-dimensional coordinate;

$\beta$ : volumetric coefficient of thermal expansion;

$\bar{\beta}$ : volumetric coefficient of expansion with concentration;

$\mu$ : viscosity;

$\rho$ : density;

$\theta$ : non-dimensional temperature;

$\kappa$ : thermal conductivity;

$v$ : kinematic viscosity. 
Appendix

$$
\begin{aligned}
& N_{1}=\frac{Q_{1} b}{K \sqrt{a S_{c}}}, \quad N_{2}=-\frac{Q_{1}}{a K S_{c}}, d=\frac{K S_{c}}{S_{c}-1}, a=\frac{1+N_{r}}{P_{r}}, \quad b=\frac{K S_{c}}{S_{c}-\frac{1}{a}} \\
& M_{1}=E_{10}-\frac{E_{5}}{\sqrt{S_{c}}}, M_{2}=E_{6}-\frac{E_{4}}{\sqrt{S_{c}}}, M_{3}=E_{8}-\frac{E_{1}}{\sqrt{S_{c}}}, M_{4}=E_{7}-\frac{E_{2}}{\sqrt{S_{c}}}, \\
& M_{5}=E_{9}-\frac{E_{3}}{\sqrt{S_{c}}}, M_{6}=P_{5}-P_{12}, M_{7}=P_{6}-P_{13}, M_{8}=P_{7}-P_{14} \text {, } \\
& M_{9}=P_{1}-P_{8}, M_{10}=P_{3}-P_{10}, M_{11}=P_{2}-P_{9}, M_{12}=P_{4}-P_{11}, \\
& E_{1}=-\frac{1}{2 i b^{3 / 2}(i \sqrt{b}+\sqrt{a})}, \quad E_{2}=-\frac{1}{2 i b^{3 / 2}(i \sqrt{b}-\sqrt{a})}, \quad E_{3}=\frac{1}{a(a+b)}, E_{4}=-\frac{1}{b \sqrt{a}}, \\
& E_{5}=-E_{1}-E_{2}-E_{3}, E_{6}=-\frac{\sqrt{K}}{b \sqrt{a}}, E_{7}=-\frac{\sqrt{K-b}}{2 i b^{3 / 2}(i \sqrt{b}-\sqrt{a})}, \\
& E_{8}=-\frac{\sqrt{K-b}}{2 i b^{3 / 2}(i \sqrt{b}+\sqrt{a})}, E_{9}=\frac{\sqrt{K+a}}{a(a+b)}, E_{10}=-E_{8}-E_{7}-E_{9}, \\
& P_{1}=-\frac{\sqrt{K S_{c}}}{b \sqrt{a}}, P_{2}=\frac{\sqrt{(K-b) S_{c}}}{2 i b^{2} \sqrt{b}(i \sqrt{b}-\sqrt{a})}, P_{3}=\frac{\sqrt{(K-b) S_{c}}}{2 i b^{2} \sqrt{b}(i \sqrt{b}+\sqrt{a})}, \\
& P_{4}=\frac{\sqrt{(K+a) S_{c}}}{a^{2}(a+b)}, P_{5}=-P_{2}-P_{3}-P_{4}, P_{6}=\sqrt{a} P_{5}+\sqrt{a} P_{3}+i \sqrt{b} P_{3}+\sqrt{a} P_{2}-i \sqrt{b} P_{2} \text {, } \\
& P_{7}=-b P_{5}+\sqrt{a} P_{6}-i \sqrt{a b} P_{3}+i \sqrt{a b} P_{2}-b P_{4}, P_{8}=-\frac{1}{b \sqrt{a}}, P_{9}=\frac{1}{2 i b^{2} \sqrt{b}(i \sqrt{b}-\sqrt{a})}, \\
& P_{10}=\frac{1}{2 i b^{2} \sqrt{b}(i \sqrt{b}+\sqrt{a})}, P_{11}=\frac{1}{a^{2}(a+b)}, P_{12}=-P_{10}-P_{9}-P_{11}, \\
& P_{13}=\sqrt{a} P_{12}+\sqrt{a} P_{10}+i \sqrt{b} P_{10}+\sqrt{a} P_{9}-i \sqrt{b} P_{9}, P_{14}=-b P_{12}+\sqrt{a} P_{13}-i \sqrt{a b} P_{10}+i \sqrt{a b} P_{9}-b P_{11} \text {, } \\
& C_{1}=\frac{G_{r} Q_{1} b d}{a K^{2} S_{c}^{2}}, \quad C_{2}=\frac{G_{r}}{1-a}, C_{3}=\frac{G_{r} Q_{1} a b}{K S_{c}(1-a) \sqrt{a}}, C_{4}=\frac{G_{m}}{K S_{c}} .
\end{aligned}
$$

